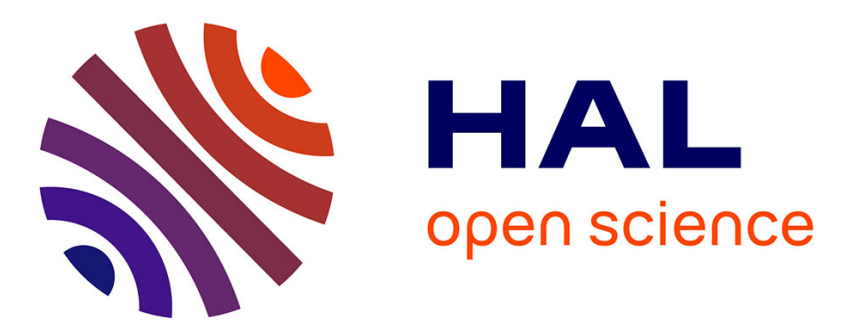

\title{
Novel classifier orthologs of bovine and human oocytes matured in different melatonin environments
}

Thanida Sananmuang, Denis Puthier, Catherine Nguyen, Kaj Chokeshaiusaha

\section{To cite this version:}

Thanida Sananmuang, Denis Puthier, Catherine Nguyen, Kaj Chokeshaiusaha. Novel classifier orthologs of bovine and human oocytes matured in different melatonin environments. Theriogenology, 2020, 156, pp.82-89. 10.1016/j.theriogenology.2020.06.029 . hal-03015699

\section{HAL Id: hal-03015699 \\ https://hal-amu.archives-ouvertes.fr/hal-03015699}

Submitted on 20 Nov 2020

HAL is a multi-disciplinary open access archive for the deposit and dissemination of scientific research documents, whether they are published or not. The documents may come from teaching and research institutions in France or abroad, or from public or private research centers.
L'archive ouverte pluridisciplinaire HAL, est destinée au dépôt et à la diffusion de documents scientifiques de niveau recherche, publiés ou non, émanant des établissements d'enseignement et de recherche français ou étrangers, des laboratoires publics ou privés. 
1 Novel Classifier Orthologs Of Bovine And Human Oocytes Matured In Different Melatonin Environments

2

5 *Corresponding author: Kaj Chokeshaiusaha

6 E-mail: kaj.chk@gmail.com

7

$8 \quad{ }^{1}$ Rajamangala University of Technology Tawan-OK, Faculty of Veterinary Medicine, Chonburi, Thailand.

$9 \quad{ }^{2}$ Aix-Marseille Université, INSERM UMR 1090, TAGC, Marseille, France.

10

11 Running title: Novel classifier orthologs for different melatonin environment of oocytes

12

13 Research funding: Thailand Research Fund (TRF) through New Research Scholar Programme (Grant No.

14 TRG5880003) 
15 Title of the manuscript: Novel classifier orthologs of bovine and human oocytes matured in different melatonin environments

\section{Abstract}

It has been demonstrated that melatonin influences the developmental competence of both in vivo and in vitro matured oocytes. It modulates oocyte-specific gene expression patterns among mammalian species. Due to differences among study systems, the identification of the classifier orthologs - the homologous genes related among mammals that could universally categorize oocytes matured in environments with varied melatonin levels is still limitedly studied. To gain insight into such orthologs, cross-species transcription profiling meta-analysis of in vitro matured bovine oocytes and in vivo matured human oocytes in low and high melatonin environments was demonstrated in the current study. RNA-Seq data of bovine and human oocytes were retrieved from the Sequence Read Archive database and pre-processed. The used datasets of bovine oocytes obtained from culturing in the absence of melatonin and human oocytes from old patients were regarded as oocytes in the low melatonin environment (Low). Datasets from bovine oocytes cultured in 10-9 M melatonin and human oocytes from young patients were considered as oocytes in the high melatonin environment (High). Candidate orthologs differentially expressed between Low and High melatonin environments were selected by a linear model, and were further verified by Zero-inflated regression analysis. Support Vector Machine (SVM) was applied to determine the potentials of the verified orthologs as classifiers of melatonin environments. According to the acquired results, linear model analysis identified 284 candidate orthologs differentially expressed between Low and High melatonin environments. Among them, only 15 candidate orthologs were verified by Zero-inflated regression analysis $(F D R \leq 0.05)$. Utilization of the verified orthologs as classifiers in SVM resulted in the precise classification of oocyte learning datasets according to their melatonin environments (Misclassification rates < 0.18, area under curves $>0.9)$. In conclusion, the cross-species RNA-Seq meta-analysis to identify novel classifier orthologs of matured oocytes under different melatonin environments was successfully demonstrated in this study- delivering candidate orthologs for future studies at biological levels. Such verified orthologs might provide valuable evidence about melatonin sufficiency in target oocytes- by which, the decision on melatonin supplementation could be implied.

Keywords: Classifier orthologs; Melatonin; Oocytes; Cross-species RNA-Seq meta-analysis 


\section{Introduction}

in vitro embryo production (IVP) is regarded as a well-established assisted reproductive technology (ART) in both humans and animal species [1] $\square$. in vitro oocyte maturation (IVM) is one of the multiple steps of IVP that the oocytes are recovered directly from the follicles and are cultured in a specific condition. Despite its advancement, incomparable in vitro and in vivo environment is still a major problem contributing to the success of IVM performance [2,3] $\square$. During in vivo oocyte development, several delicate substances and hormones are locally secreted in follicular fluid by ovarian and granulosa cells to nourish oocytes. This includes melatonin which was widely studied for its contribution to oocyte quality [2-5]. Melatonin was most recognized for its scavenging function on reactive oxygen species (ROS). Since ROS play a major role in impairing oocyte development [6,7], the lack of melatonin in conventional in vitro culture environments has contributed to ROSinduced poor oocyte quality in several mammals $[3,8,9] \square$.

Several factors also affect in vivo oocyte development. This includes reproductive aging-one of the most common causes of female infertility. It has been reported that reproductive aging induces a variety of physiological aberrations withering oocyte quality [9-12] $\square$. One of them is melatonin deprivation, caused by reduced melatonin production from aging granulosa and ovarian cells. Melatonin deprivation was hereby blamed as a major contributor to aging oocyte's incompetency [2,9] $\square$. Aging also results in other diverse hormonal imbalances and assorted physiological changes $[9,13] \square$. All these factors represent complex negative effects during in vivo oocyte aging. Therefore, it has been difficult to exclusively determine the melatonin deprivation effects on the oocyte during in vivo development in such circumstances. By means of this, it was difficult to exclusively determine the melatonin deprivation effect on in vivo oocyte in such circumstances.

Interestingly, similar aging aberrance also occurred with in vitro cultured oocytes in a time-dependent manner. in vitro-aging phenomenon also included ROS-induced quality deterioration due to the absence of melatonin in the conventional culture environment $[2-5,9,11] \square$. In agreement with the protective effects of in vivo melatonin produced by ovarian follicles, in vitro melatonin supplement was evidenced to restore the oocyte developmental competency in several species [3-5,9] $\square$. Unlike in vivo-aging condition divided effects of in vitro melatonin deprivation should be conveniently observed due to the simplicity of the culture environment. Through this, analysis of crossed effects between in vitro and in vivo melatonin deprivation due to aging should hereby contribute a novel insight into the universal melatonin effects independent from other factors.

With comparable reproductive physiology and embryogenesis, cattle are regarded as excellent study models for human IVP $[10,14] \square$. Employing this, novel transcriptomic technologies including RNA sequencing (RNA-Seq) have been continuously applied with oocyte samples obtained from both species for studying their 
universal transcriptome's regulation. However, the application of standard RNA-Seq or bulk RNA-Seq with mammalian oocytes was not practical due to low oocyte numbers for cDNA library preparation $[15,16] \square$. Moreover, oocytes were heterogeneous populations, and should thus not represented with average results. Since cell-to-cell variations are common among isolated oocytes, loss of real features among them in bulk analysis could bring about considerable bias in the analysis [15].

Recently, low-input and single-cell RNA sequencing (RNA-Seq) methods have been implemented with oocytes acquired from various mammals $[3,15,17] \square$. Since such technologies allow robust transcript profiling analysis down to a single cell, they enable researchers to explore biological variations among oocytes with reduced bias $[3,16,18] \square$. Interestingly, an increase of such data among a variety of mammalian species even allowed cross-species meta-analysis of oocytes. Since cross-species transcription profiling meta-analysis of oocytes could imply universal orthologs regulated among the species in the environment of interest [19], implement of such analysis with RNA-Seq data of oocytes obtained from different in vitro and in vivo melatonin environments should imply us insight into such orthologs, as well.

In several transcription profiling studies, genomic classification or subtyping of unknown samples can be archived by various machine learning algorithms. Support vector machine (SVM) is a robust learning model widely implemented on a genome-wide scale to prioritize orthologs for sample classification [20,21] $\square$. Novel classifier orthologs were identified by SVM in several topics including aging, cancers, infections, and metabolic disorders [22-25]. Regardless of its flexibility, SVM application in oocyte study was still limitedly demonstrated. It was noteworthy that the integration of SVM with cross-species meta-analysis of different melatonin environments could thus help us determining novel classifier orthologs for evaluating the melatonin sufficiency of oocytes. In other words, such cognition would preliminarily assess the beneficial effects of melatonin supplement on target oocytes presented in environments of interest.

To gain insight into universal classifier orthologs for melatonin environments, cross-species transcription profiling meta-analysis of in vitro matured bovine oocytes and in vivo matured human oocytes in low and high melatonin environments was performed in the current study. The pooled cDNA library based on human-bovine orthologs was constructed with bias correction. With restricted verification procedures and SVM classification, the universal classifier orthologs for melatonin environments of oocytes were successfully implied for the first time in this study.

\section{Materials and methods}




\subsection{Sample datasets}

RNA-Seq data of bovine and human oocytes were retrieved from the sequence read archive (SRA) database (https://www.ncbi.nlm.nih.gov/sra) (Table 1). Both bovine and human RNA-Seq data were presented in NCBI with accession numbers GSE122738 [3] $\square$ and GSE125300 [26] $\square$ (https://www.ncbi.nlm.nih.gov/geo/). The sources of bovine [3] $\square$ and human oocytes [26] $\square$ utilized for data preparation were summarized in Table 1 . In brief, bovine cumulus- oocyte complexes (COCs) were collected from slaughterhouses and cultured in the presence (In_vitro_MII+Mela) or absence (In_vitro_MII) of $10^{-9} \mathrm{M}$ melatonin for 24 hours, respectively. Metaphase II (MII) oocytes were isolated from COCs and divided into 6 samples—each containing 7 oocytes of their groups. On the contrary, each human MII oocyte sample was retrieved directly from single follicles of young (In_vivo_young_MII), or old (In_vivo_old_MII) patients by ultrasound-guided technique. In this study, the term-melatonin environment was used to infer the amount of melatonin in the environment surrounding the target oocyte samples. Through this, the bovine oocytes matured in the absence of melatonin supplement, and human oocytes acquired from old patients were regarded as oocytes in the low melatonin environment. On the other hand, bovine oocytes matured in the presence of melatonin supplement, and human oocytes acquired from young patients were considered as oocytes in the high melatonin environment.

\subsection{Data pre-processing}

Data pre-processing was performed with the process similar to that described in our previous study [27] $\square$. In brief, all sequences were trimmed to remove contaminated adapter sequences and unqualified sequences (length $<25$ nucleotides and mean Phred score $<25$ ). Duplicated sequences removal, sequence alignments (>85\%), and GC base-balance bias correction were accomplished prior to gene-level counting. In this study, the read counts were normalized by the trimmed mean of M-values (TMM). bovine-human orthologs were assigned with the previously reported procedure [19] $\square$. Data quality control was carried out [28] $\square$ along with cell-cycle effect removal to minimize the bias from within-cell-type heterogeneity [29] $\square$. Count read numbers of all samples were $>14 \times 10^{-6}$, and none of them was prone to apoptosis (Fraction of mitochondrial reads $<1.5 \%$ ). The mutual nearest neighbors (MNN) in ortholog expression space was identified for cross-species batch correction [30] $\square$ of pooled cDNA library created from bovine and human oocyte count data using 'scater' package [31] $\square$. 
Candidate orthologs for oocyte sample categorization by melatonin environment were selected using procedures as described in our previous study [19]. In brief, candidate bovine-human orthologs differentially expressed between oocytes in low and high melatonin environments were selected from 1,000 learning datasets using the linear model and empirical Bayes methods. In this study, we consider differentially expressed orthologs with importance value $\geq 2.5$. Only orthologs declared significant both by intra-species and cross-species analyses would be selected as candidate orthologs. With expression values of the candidate orthologs, Partitioning around medoids (PAM) clustering (2-4 clusters) was performed, and clustering validation was determined by Connectivity, Dunn, and Silhouette scores.

\subsection{Verification of the candidate orthologs}

Concerning about unrealistic mean-variance relationship produced by the batch-corrected data, the candidate orthologs were verified by Zero-inflated regression analysis with proper model matrix using raw count data to avoid the bias from the batch correction process with 'MAST' package [32] $\square$. The same criteria of candidate ortholog selection was applied-by which the verified candidate orthologs must be differentially expressed between low and high melatonin environments both by intra-species and cross-species analyses (FDR $\leq 0.05)$

\subsection{Oocyte sample classification by Support Vector Machine}

To determine the potential of the verified candidate orthologs as classifiers for melatonin environments, Support Vector Machine (SVM) with linear kernel classifier was performed. Different training data were generated by 5 -fold cross-validation, Bootstrap, and Monte-Carlo-cross-validation strategies-1,000 iterations for each strategy. All procedures could be archived by class prediction functions using the 'CMA' package [20] $\square$.

\subsection{Data visualization}

Heatmap illustrating candidate orthologs was drawn using the “ComplexHeatmap” package [33] $\square$. The silhouette width values acquired from PAM clustering were illustrated by the bar plot drawn by the 'ggplot2' packages. A bubble plot manifesting $-\log _{10}$ FDR values and $\log _{2}$ fold-change values of the verified candidate orthologs was drawn using the 'ggplot2' package. Probability plots and Receiver operating characteristic (ROC) curves were drawn to illustrate the classification accuracy of the verified candidate orthologs by the 'ggplot2' package. 


\section{Results}

3.1 Oocyte samples could be clustered by their melatonin environments using the candidate orthologs as features.

Analysis of bias-corrected data by linear model revealed 284 candidate orthologs differentially expressed between oocytes in low and high melatonin environments (importance value $\geq 2.5$ ). According to PAM clustering results based on expressions of these candidate orthologs (Table 2 and Fig 1), oocyte samples were correctly categorized into 2 clusters corresponding to their melatonin environments regardless of the species (Fig 2).

3.2 All verified candidate orthologs were significantly expressed in oocytes in the high melatonin environment. Zero-inflated regression analysis revealed 158 orthologs differentially expressed between oocytes in low and high melatonin environments (FDR $\leq 0.05)$. However, only 15 orthologs were found intersected with the candidate orthologs acquired from the previous selections-AAMP, ADM, ATP6V0A2, BCL7B, CYB561D2, ENDOV, F2RL1, KIAA0586, LPCAT4, NR1H3, PLEKHM1, SLC47A1, SYT11, RFT1, and MRPS18C (Fig 3). While all oocytes in the high melatonin environment expressed these orthologs higher than those in the low environment, only ADM, CYB561D2, ENDOV, KIAA0586, NR1H3, PLEKHM1, SLC47A1, SYT11, RFT1, and MRPS18C rendered substantial fold-change values $(\geq 1.5)$. According to our review of their biological functions, such orthologs were associated with a variety of cell metabolisms (Table 3).

3.3 Verified candidate orthologs were potential classifiers for the melatonin environments of oocytes.

The verified candidate orthologs were used as classifier orthologs in SVM performance. Consistent results were acquired among learning datasets — by which all of them rendered Misclassification rates $<0.18$, and their ROCs had Area Under Curves (AUCs) > 0.9 (Fig 4).

\section{Discussion}

A cross-species transcription profiling meta-analysis of in vitro matured bovine oocyte data and in vivo matured human oocyte data to identify classifier orthologs for different melatonin environments was performed in this study. Despite the discreet procedures manifested, some limitations in result interpretation should be greatly concerned to avoid over-assumption. As described in the methodology, the in vitro and in vivo matured oocyte samples used in this study were procured from different studies with limited information about them. For 
instance, melatonin concentrations in follicles containing in vivo matured human oocytes were not indicated, while the concentrations were clearly stated in bovine oocytes' culture systems. Undetermined differences in melatonin concentrations between such environments should reduce the sensitivity of differential analysis in this study. With more available in vivo matured oocyte profiling data with well-defined melatonin environments in the future, the list of classifier orthologs could be improved for better generalization.

As expected, our previously reported procedures [19] $\square$ were also applicable to the selection of candidate orthologs differentially expressed between low and high melatonin environments (Fig 2 and Table 2). While these orthologs could be used to annotate essential melatonin functions on in vivo and in vitro matured oocytes, this study aimed to identify the most reliable classifier orthologs among them. Due to such delegation, we further verified the candidate orthologs with the zero-inflated regression test to reduce the mean-variance bias (Fig 3).

Encoded products of the verified candidate orthologs were associated with in various oocyte metabolisms (Table 3). In detail, ADM encoded an angiogenic factor, adrenomedullin-an important vasorelaxant required during folliculogenesis and oocyte maturation processes [34,35]. CYB561D2 encoded a member of trans-membrane (TM) protein family involving stress defense and iron metabolism [36]. ENDOV encoded Endoribonuclease V—an enzyme involving with the DNA repair process of oocyte [37] $\square$. KIAA0586 encoded a conserved centrosomal protein required for sonic hedgehog/SHH signaling during the oocyte developmental process [38]. NR1H3-activating ligands helped with oocyte meiosis resumption [39] $\square$. PLEKHM1 could regulate the autophagy process which was crucial in the regulation of oocyte metabolism [4042] $\square$. SLC47A1 encoded an extrusion protein for drug and toxic metabolites [43] $\square$. SYT11 encoded synaptotagmin-11 for exocytosis and endocytosis regulation [44] $\square$. RFT1 encoded a major folate transporter [45], which helped to accumulate folate for normal oogenesis and early embryogenesis [46] $\square$. Lastly, MRPS18C encoded a mitochondrial ribosomal protein and thus was required for other mitochondrial protein syntheses $[47] \square$.

The high melatonin environment was shown to enhance expressions of all verified candidate orthologs (Fig 3 and Table 3). Supporting this notice, enhanced expression of KIAA0586 by melatonin was already notified in the other study [8]. Since functions of these orthologs were strongly associated with qualified oocyte development as previously mentioned, their diminished expressions in the low melatonin environment should thus adversely affect the oocyte development (Table 3). As far as we know, shreds of evidence of enhanced functions of most verified orthologs by melatonin were still not warranted in mammalian oocytes - by which future studies to confirm such effects were encouraged. 
In the current study, the classifying potential of the verified orthologs was determined by SVM to assess their reliability and reproducibility. According to the result, low misclassification scores and high AUCs manifested among tested learning datasets (Fig 4) hereby indicated such orthologs as potential classifiers for melatonin environments. Though utilization of cross-species data in this study partially suggested such classifier orthologs to be universally applicable among mammalian oocytes, further confirmation in other mammals with known melatonin environments were still required. Since overall expression levels of acquired classifier orthologs could imply sufficiency of melatonin, we, on a certain level proposed them as novel parameters to provide some clues about beneficial effects of melatonin supplement on target oocytes in any culture environments of interest. Such knowledge greatly relieved burdens for researchers to screen for universal melatonin effects on all ortholog expressions which were not possible by the conventional approach. It should however be noted that these classifier orthologs might not only be specific to the melatonin environment and thus required further classification tests with other possible oocyte environments.

\section{Conclusion}

Novel classifier orthologs of oocytes matured under low and high melatonin environments were identified by cross-species transcription profiling meta-analysis in this study. Such an outcome not only offered us future melatonin research topics to evaluate classifying potentials of the verified orthologs at biological levels but also the complete process to acquire them from available RNA-Seq data. We would like to note that the analytical procedures demonstrated in the current study could be applied with other oocyte environments of interest - by which identification of other novel parameters of other oocyte culture environments was possible.

\section{Conflict of interest}

We certify that there is no conflict of interest with any financial organization regarding the material and methods discussed in the manuscript.

\section{Acknowledgments}

The current study was fully supported by the Thailand Research Fund (TRF) through New Research Scholar Program (Grant No. TRG5880003). We would like to show our gratitude to the Office of Academic Resource and Information Technology (ARiT), Rajamangala University of Technology Tawan-OK (RMUTTO) for the contribution of the server computer for the burden analysis. 


\section{References}

[1] Wang J, Sauer M V. in vitro fertilization (IVF): A review of 3 decades of clinical innovation and technological advancement. Ther Clin Risk Manag 2006;2:355-64. https://doi.org/10.2147/tcrm.2006.2.4.355.

[2] Zhang M, Lu Y, Chen Y, Zhang Y, Xiong B. Insufficiency of melatonin in follicular fluid is a reversible cause for advanced maternal age-related aneuploidy in oocytes. Redox Biol 2020;28:101327. https://doi.org/10.1016/j.redox.2019.101327.

[3] An Q, Peng W, Cheng Y, Lu Z, Zhou C, Zhang Y, et al. Melatonin supplementation during in vitro maturation of oocyte enhances subsequent development of bovine cloned embryos. J Cell Physiol 2019;234:17370-81. https://doi.org/10.1002/jcp.28357.

[4] Do LTK, Shibata Y, Taniguchi M, Nii M, Nguyen T V., Tanihara F, et al. Melatonin Supplementation During in vitro Maturation and Development Supports the Development of Porcine Embryos. Reprod Domest Anim 2015;50:1054-8. https://doi.org/10.1111/rda.12607.

[5] Soto-Heras S, Catalá MG, Roura M, Menéndez-Blanco I, Piras AR, Izquierdo D, et al. Effects of melatonin on oocyte developmental competence and the role of melatonin receptor 1 in juvenile goats. Reprod Domest Anim 2019;54:381-90. https://doi.org/10.1111/rda.13378.

[6] El-Raey M, Geshi M, Somfai T, Kaneda M, Hirako M, Abdel-Ghaffar AE, et al. Evidence of melatonin synthesis in the cumulus oocyte complexes and its role in enhancing oocyte maturation in vitro in cattle. Mol Reprod Dev 2011;78:250-62. https://doi.org/10.1002/mrd.21295.

[7] Jing T, Shile S, Sun Y, Li H, Li WP, Cong Z, et al. Melatonin levels in follicular fluid as markers for IVF outcomes and predicting ovarian reserve. Reproduction 2017;153:443-51. https://doi.org/10.1530/REP$16-0641$.

[8] Lee S, Jin JX, Taweechaipaisankul A, Kim GA, Ahn C, Lee BC. Melatonin influences the sonic hedgehog signaling pathway in porcine cumulus oocyte complexes. J Pineal Res 2017;63. https://doi.org/10.1111/jpi.12424.

[9] Igarashi H, Takahashi T, Nagase S. Oocyte aging underlies female reproductive aging: biological mechanisms and therapeutic strategies. Reprod Med Biol 2015;14:159-69. https://doi.org/10.1007/s12522-015-0209-5.

[10] Malhi PS, Adams GP, Singh J. A bovine model to study reproductive aging. Biol Reprod 2005;73:45-53. https://doi.org/10.1095/biolreprod.104.038745.

[11] Malhi PS, Adams GP, Mapletoft RJ, Singh J. Oocyte developmental competence in a bovine model of reproductive aging. Reproduction 2007;134:233-9. https://doi.org/10.1530/REP-07-0021.

[12] Thouas GA, Trounson AO, Jones GM. Effect of Female Age on Mouse Oocyte Developmental Competence Following Mitochondrial Injury1. Biol Reprod 2005;73:366-73. https://doi.org/10.1095/biolreprod.105.040956.

[13] George K, Kamath M. Fertility and age. J Hum Reprod Sci 2010;3:121-3. https://doi.org/10.4103/09741208.74152 .

[14] Hansen PJ. Realizing the promise of IVF in cattle - An overview. Theriogenology 2006;65:119-25. https://doi.org/10.1016/j.theriogenology.2005.09.019.

[15] Brayboy LM, Wessel GM. The double-edged sword of the mammalian oocyte - advantages, drawbacks and approaches for basic and clinical analysis at the single cell level. Mol Hum Reprod 2015;22:200-7. https://doi.org/10.1093/molehr/gav064. 
[16] Chambers DC, Carew AM, Lukowski SW, Powell JE. Transcriptomics and single-cell RNA-sequencing. Respirology 2019;24:29-36. https://doi.org/10.1111/resp.13412.

[17] Liu XM, Wang YK, Liu YH, Yu XX, Wang PC, Li X, et al. Single-cell transcriptome sequencing reveals that cell division cycle 5-like protein is essential for porcine oocyte maturation. J Biol Chem 2018;293:1767-80. https://doi.org/10.1074/jbc.M117.809608.

[18] Chitwood JL, Burruel VR, Meyers SA, Ross PJ. 131 RNA-Seq TRANSCRIPTOME PROFILING OF INDIVIDUAL RHESUS MACAQUE OOCYTES AND PRE-IMPLANTATION EMBRYOS. Reprod Fertil Dev 2014;26:179. https://doi.org/10.1071/rdv26n1ab131.

[19] Chokeshaiusaha K, Puthier D, Nguyen C, Sananmuang T. Construction of pooled oocyte expression profiles of rhesus monkey and mouse for concurrent meta-analyses. Thai J Vet Med 2015;45:523-33.

[20] Slawski M, Daumer M, Boulesteix AL. CMA - A comprehensive Bioconductor package for supervised classification with high dimensional data. BMC Bioinformatics 2008;9. https://doi.org/10.1186/14712105-9-439.

[21] Fernandes de Mello R, Antonelli Ponti M, Fernandes de Mello R, Antonelli Ponti M. Introduction to Support Vector Machines. Mach. Learn., 2018, p. 163-226. https://doi.org/10.1007/978-3-319-949895 4.

[22] Huang S, Nianguang CAI, Penzuti Pacheco P, Narandes S, Wang Y, Wayne XU. Applications of support vector machine (SVM) learning in cancer genomics. Cancer Genomics and Proteomics 2018;15:41-51. https://doi.org/10.21873/cgp.20063.

[23] Wang HY, Chang SC, Lin WY, Chen CH, Chiang SH, Huang KY, et al. Machine learning-based method for obesity risk evaluation using single-nucleotide polymorphisms derived from next-generation sequencing. J Comput Biol 2018;25:1347-60. https://doi.org/10.1089/cmb.2018.0002.

[24] Kumar A, Jeya Sundara Sharmila D, Singh S. SVMRFE based approach for prediction of most discriminatory gene target for type II diabetes. Genomics Data 2017;12:28-37. https://doi.org/10.1016/j.gdata.2017.02.008.

[25] Arabfard M, Ohadi M, Rezaei Tabar V, Delbari A, Kavousi K. Genome-wide prediction and prioritization of human aging genes by data fusion: A machine learning approach. BMC Genomics 2019;20. https://doi.org/10.1186/s12864-019-6140-0.

[26] Zhang JJ, Liu X, Chen L, Zhang S, Zhang X, Hao C, et al. Advanced maternal age alters expression of maternal effect genes that are essential for human oocyte quality. Aging (Albany NY) 2020. https://doi.org/10.18632/aging.102864.

[27] Chokeshaiusaha K, Puthier D, Nguyen C, Sudjaidee P, Sananmuang T. Factor Analysis for Bicluster Acquisition (FABIA)revealed vincristine-sensitive transcript pattern of canine transmissible venereal tumors. Heliyon 2019;5:e01558. https://doi.org/10.1016/j.heliyon.2019.e01558.

[28] Luecken MD, Theis FJ. Current best practices in single- cell RNA- seq analysis: a tutorial. Mol Syst Biol 2019;15. https://doi.org/10.15252/msb.20188746.

[29] Barron M, Li J. Identifying and removing the cell-cycle effect from single-cell RNA-Sequencing data. Sci Rep 2016;6. https://doi.org/10.1038/srep33892.

[30] Shafer MER. Cross-Species Analysis of Single-Cell Transcriptomic Data. Front Cell Dev Biol 2019;7. https://doi.org/10.3389/fcell.2019.00175.

[31] Haghverdi L, Lun ATL, Morgan MD, Marioni JC. Batch effects in single-cell RNA-sequencing data are corrected by matching mutual nearest neighbors. Nat Biotechnol 2018;36:421-7. https://doi.org/10.1038/nbt.4091. 
[32] Finak G, McDavid A, Yajima M, Deng J, Gersuk V, Shalek AK, et al. MAST: A flexible statistical framework for assessing transcriptional changes and characterizing heterogeneity in single-cell RNA sequencing data. Genome Biol 2015;16. https://doi.org/10.1186/s13059-015-0844-5.

[33] Gu Z, Eils R, Schlesner M. Complex heatmaps reveal patterns and correlations in multidimensional genomic data. Bioinformatics 2016;32:2847-9. https://doi.org/10.1093/bioinformatics/btw313.

[34] Malamitsi-Puchner A, Sarandakou A, Baka SG, Tziotis J, Rizos D, Hassiakos D, et al. Concentrations of angiogenic factors in follicular fluid and oocyte-cumulus complex culture medium from women undergoing in vitro fertilization: Association with oocyte maturity and fertilization. Fertil Steril 2001;76:98-101. https://doi.org/10.1016/S0015-0282(01)01854-4.

[35] Manau D, Balasch J, Jiménez W, Fábregues F, Civico S, Casamitjana R, et al. Follicular fluid concentrations of adrenomedullin, vascular endothelial growth factor and nitric oxide in IVF cycles: Relationship to ovarian response. Hum Reprod 2000;15:1295-9. https://doi.org/10.1093/humrep/15.6.1295.

[36] Asard H, Barbaro R, Trost P, Bérczi A. Cytochromes b561: Ascorbate-mediated trans-membrane electron transport. Antioxidants Redox Signal 2013;19:1026-35. https://doi.org/10.1089/ars.2012.5065.

[37] Cao W. Endonuclease V: An unusual enzyme for repair of DNA deamination. Cell Mol Life Sci 2013;70:3145-56. https://doi.org/10.1007/s00018-012-1222-z.

[38] Lee S, Jin JX, Taweechaipaisankul A, Kim GA, Ahn C, Lee BC. Sonic hedgehog signaling mediates resveratrol to improve maturation of pig oocytes in vitro and subsequent preimplantation embryo development. J Cell Physiol 2018;233:5023-33. https://doi.org/10.1002/jcp.26367.

[39] Steffensen KR, Robertson K, Gustafsson JA, Andersen CY. Reduced fertility and inability of oocytes to resume meiosis in mice deficient of the Lxr genes. Mol Cell Endocrinol 2006;256:9-16. https://doi.org/10.1016/j.mce.2006.03.044.

[40] Sagrillo-Fagundes L, Bienvenue-Pariseault J, Vaillancourt C. Melatonin: The smart molecule that differentially modulates autophagy in tumor and normal placental cells. PLoS One 2019;14. https://doi.org/10.1371/journal.pone.0202458.

[41] Lin FH, Zhang WL, Li H, Tian XD, Zhang J, Li X, et al. Role of autophagy in modulating postmaturation aging of mouse oocytes article. Cell Death Dis 2018;9. https://doi.org/10.1038/s41419-0180368-5.

[42] Shen XH, Jin YX, Liang S, Kwon JW, Zhu JW, Lei L, et al. Autophagy is required for proper meiosis of porcine oocytes maturing in vitro. Sci Rep 2018;8. https://doi.org/10.1038/s41598-018-29872-y.

[43] Yonezawa A, Inui KI. Importance of the multidrug and toxin extrusion MATE/SLC47A family to pharmacokinetics, pharmacodynamics/toxicodynamics and pharmacogenomics. Br J Pharmacol 2011;164:1817-25. https://doi.org/10.1111/j.1476-5381.2011.01394.x.

[44] Wang C, Wang Y, Hu M, Chai Z, Wu Q, Huang R, et al. Synaptotagmin- 11 inhibits clathrin- mediated and bulk endocytosis. EMBO Rep 2016;17:47-63. https://doi.org/10.15252/embr.201540689.

[45] Umapathy NS, Gnana-Prakasam JP, Martin PM, Mysona B, Dun Y, Smith SB, et al. Cloning and functional characterization of the proton-coupled electrogenic folate transporter and analysis of its expression in retinal cell types. Investig Ophthalmol Vis Sci 2007;48:5299-305. https://doi.org/10.1167/iovs.07-0288.

[46] Meredith M, MacNeil AH, Trasler JM, Baltz JM. Growing Mouse Oocytes Transiently Activate Folate Transport via Folate Receptors As They Approach Full Size1. Biol Reprod 2016;94. https://doi.org/10.1095/biolreprod.115.137687. 
387 [47] Van Blerkom J. Mitochondrial function in the human oocyte and embryo and their role in developmental competence. Mitochondrion 2011;11:797-813. https://doi.org/10.1016/j.mito.2010.09.012. 
390 Figure 1. Silhouette plot of PAM clustering. The average silhouette width was 0.395 when dividing oocyte samples into 2 clusters.

Figure 2. Heatmap of PAM clustering results. Rows of heatmap represented scaled expression values of the candidate orthologs clustered by k-means clustering, while the columns represented oocyte samples clustered by PAM.

Figure 3. Bubble plot of the verified candidate orthologs. The blue and red colors indicated scaled expression levels of target orthologs expressed by oocytes in the low melatonin environment comparing to those obtained from the high melatonin environment. The color intensities were corresponded to the $-\log _{10}$ FDR values, while the dot sizes were proportional to the absolute $\log _{2}$ fold-change values of differentially expressed orthologs.

401

Figure 4. Predicted probability plots and ROC curves. Predicted probabilities plots were displayed by colored dots, where red dots corresponded to class 0 - low melatonin environment, and green dots for class 1 - high melatonin environment. The misclassification rate acquired from each learning dataset was shown under the plot (5-fold cross-validation, Bootstrap, and Monte-Carlo-cross-validation). Adjacent to each predicted probability plot, the ROC curve generated from its same learning dataset was drawn with AUC value demonstrated. 